Article

\title{
Occlusal Load Considerations in Implant-Supported Fixed Restorations
}

\author{
Cesare D'Amico ${ }^{1}$, Salvatore Bocchieri ${ }^{2}\left(\mathbb{D}\right.$, Sergio Sambataro ${ }^{1}\left(\mathbb{D}\right.$, Giovanni Surace ${ }^{3,4}$, \\ Chiara Stumpo ${ }^{1}$ and Luca Fiorillo ${ }^{1, * \text { CD }}$ \\ 1 Department of Biomedical and Dental Sciences, Morphological and Functional Images, University of \\ Messina, 98100 Messina, Italy; cdamico@unime.it (C.D.); ssambataro@centrodiortodonzia.it (S.S.); \\ chiarastumpo92@gmail.com (C.S.) \\ 2 Department of Medicine and Surgery, School of Medicine, University of Insubria, 21100 Varese, Italy; \\ salvo.bocchieri@gmail.com \\ 3 Clinical Analysis Laboratory “Dott. Francesco Siracusa Rizzi s.r.l.”, Via Nazionale Archi, \\ 89121 Reggio Calabria, Italy; giox84@hotmail.com \\ 4 Unit of Microbiology and Virology, North Health Center ASP 5, 89100 Reggio Calabria, Italy \\ * Correspondence: lfiorillo@unime.it; Tel.: +39-090-221-6920
}

Received: 22 July 2020; Accepted: 29 September 2020; Published: 1 October 2020

check for updates

\begin{abstract}
The advent of new technologies in the field of medicine and dentistry is creating improvements that lead clinicians to have materials and procedures able to improve patients' quality of life. The aim of this article is to evaluate occlusion load and its consequences on fixed implant-supported prosthesis. New materials have granted clinicians the possibility achieve great aesthetic results in dental prosthesis, and new procedures allow them to standardize and give precise and repeatable results, especially for the functional and long-term stability aspects of products. Some principles should be carefully evaluated and applied to every dental prosthesis; the evaluation of the forces and fitting of meso-structures to dental implants, an aspect that is often not well considered by clinicians, is the main focus of this article.
\end{abstract}

Keywords: rehabilitation; dental implant; prosthodontics; dentistry; peri-implant tissue; occlusion; TMJ

\section{Introduction}

Implant prosthesis is a prognostically favorable rehabilitation method, if supported by a correct diagnostic procedure and performed according to rigorous and standardized surgical and prosthetic protocols [1]. Although the surgical protocol has been verified and considered predictable, some failures are always reported in remote follow-ups regardless of the techniques analyzed [2]. These results justify the interest that researchers have shown in recent years in analyzing the causes that could determine dental implant failure [3-5]. The factors that appear to be the most responsible for the failures are attributable to infectious factors (peri-implantitis) and/or overload (the management of occlusal relationships) [6]. The correlation between inadequate oral hygiene and marginal bone loss is well-documented both clinically and experimentally. The correlation between overload and marginal bone loss is less clear. This is linked to the impossibility of quantifying the intensity and direction of the chewing forces that develop in the oral cavity, especially for patients who show a parafunction, and to relate them to the biomechanical abilities of the bone [7-9]. The causes of overload also include the tensions deriving from the non-passivation of the metals of the prosthetic structures that connect several implants $[10,11]$. These tensions would be capable of compromising osseointegration at the bone-implant interface and, therefore, the long-term result of prosthetic implant rehabilitation. It therefore appears necessary to analyze which pathogenetic theories and occlusal 
dynamics support bone resorption as a function of overload [12-14]. The subject of this discussion is the evaluation of the forces deriving from the failure to adapt the mesostructures which act on fixed dental implant-supported rehabilitation.

\section{Materials and Methods}

\subsection{Intraoral Force Analysis}

The decisive difference between a natural tooth and an osseointegrated implant is that the latter, by definition, does not have any type of tissue interposed between its surface and that of the bone to which it is rigidly anchored (functional ankylosis) [15]. Therefore, the forces that are exercised during chewing can generate stress at the level of the restorations on the implants, their components, and the interface between the implant and the bone which could be greater than that on natural teeth [16]. Furthermore, due to bone resorption, which occurs as a result of tooth loss, even if modern implantology is prosthetically guided, the implants are often placed in an apical and or lingual position to their natural predecessors. The point of application of the intraoral forces is thus more distant from the bone crest where the implant resides and the lever arm increases (Figure 1). As a result, load transmission situations are sometimes unfavorable from a strictly mechanical point of view. To prevent, therefore, the risks of system overload (component fracture and/or loss of osseointegration), it is important to know the types of forces that can be generated in the mouth. The forces differ according to the direction, intensity, and method of application $[17,18]$.

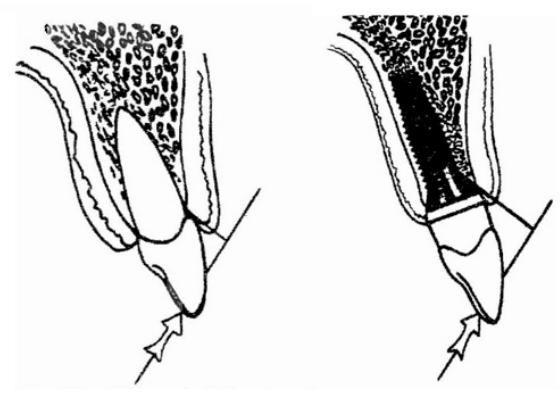

Figure 1. Peri-implant tissue reabsorbment. In the case of angled occlusal load (as shown by the arrow), teeth and dental implants respond in different ways.

\subsubsection{Axial Direction}

Axial or coaxial loading occurs when occlusal forces are exerted along the vertical axis of the implant. As with natural teeth, these are the most favorable forces, since they are distributed equally along the surface of the interface between the bone and implant. Axial forces can be tensile or compressive. The latter are to be favored, since the maximum load that the mechanical components can withstand before a failure is higher if the same components are stressed in compression rather than in tension. Clinically, a force acting in an exclusively axial direction is considered improbable, or at least a simplification of a more complex reality. In fact, each force can be decomposed into vectors with different directions and actions than the original ones.

\subsubsection{Transverse or Lateral Direction}

If the prosthesis on implants is stressed by a force acting in a transverse direction or if the axial force is applied at a point far from the axis of the implant, the implant prosthesis unit will be subject to bending or twisting-i.e., an action that tends to rotate its body (Figure 2). These transverse forces are considered less favorable than the axial compressive ones due to the potentially deleterious effects that they can have on both the components and the bone support. Ideally, any type of transverse force should be reduced or eliminated, but this is impossible. A remedy to prevent fracture could be an increase in the diameter of the implants and related components; at the same time, it would be ideal 
to insert implants as long as possible, so that, while the transverse load remains the same, the stress transferred to the bone in the apical and coronal areas will be less, since the surface on which the load is distributed is greater.

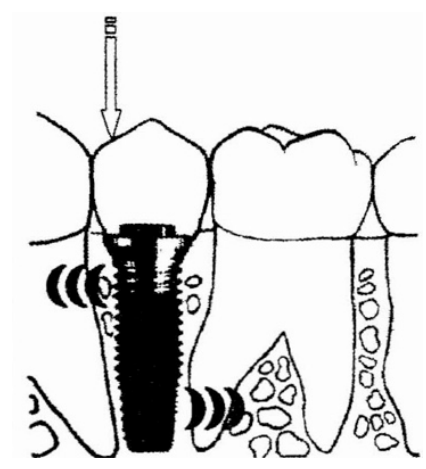

Figure 2. Peri-implant tissue force dissipation. Arrow shows the occlusal load, and the dissipation of these forces to the bone has been marked.

\subsubsection{Intensity}

Especially in the posterior sectors, the intensity of the masticatory forces recorded on implant-supported prostheses is such as to cause concern for the possible incidence of problems both mechanical (component fracture) and biomechanical (loss of osseointegration). It is therefore important that these forces are transmitted with a dynamic and in directions that do not cause an overload.

\subsubsection{Application Procedure}

Occlusal loads can be applied dynamically or statically. The first group includes all those forces generated during the usual masticatory function. In the second group, on the other hand, are the forces and stresses originating from parafunctional habits and tensions due to the insertion of prostheses that do not fit precisely and passively with the implants. For the survival of rehabilitations on implants, static loads are considered potentially more dangerous than dynamic loads.

\subsection{Biological Complications of the Overload}

In implantology, the achievement of long-lasting functional ankylosis between bone and implant is the primary objective to be targeted and the essential condition on which the outcome of the treatment depends.

Bone tissue has the ability to continuously adapt to local mechanical conditions and, for this reason, particular attention must be paid to the stresses transmitted to it by the integrated implant.

In fact, not all stimuli are equally favorable and not all determine a new bone apposition; some authors say that, in overload situations, peri-implant bone resorption can be triggered with a consequent loss of ankylosis and the formation of fibrous connective tissue between the implant and bone $[19,20]$.

The loss of osseointegration can be determined by both mechanical (loading theory) and microbiological factors, which can determine the onset of peri-implantitis. From a purely mechanical point of view, the loss of functional ankylosis must be understood as the overcoming of the ability to regulate the local homeostasis of the bone tissue, followed by the morpho-structural reorganization of the peri-implant tissue. In any case, the loss of osseointegration represents in all respects a failure of implant therapy. Functional loads above a certain threshold would therefore trigger a demolition adaptation of the implant-bone interface, which affects the entire surface. The loss of this intimate contact advances in relation to the frequency and extent of the stresses transmitted to the bone and stops only when the adaptation of the bone substrate is able to organize itself in a positive sense, 
reaching a new functional balance. Thus, a fibrous capsule is formed to replace the previously present bone tissue, arranged so as to completely envelop the implant and represented radiographically by a peri-implant radiolucency line, while on clinical examination the tissues appear to be normally structured. The loss of osseointegration can therefore be explained with plaque theory and also with loading theory. To evaluate these phenomena at a clinical level and to differentiate bone loss caused by overload from that of an infectious nature (peri-implantitis), it is necessary to identify parameters capable of causing reabsorption or failure of an etiological nature. Furthermore, the reorganization of the tissues and the subverted bone architecture can facilitate the establishment of a marginal bacterial superinfection, which can spread more easily in depth.

\subsection{Stresses and Osteointegration}

Clinical studies report that the main causes of implant loss are the accumulation of bacterial plaque, the presence of type IV bone, and overload. The latter can be defined as the existence of destructive stress levels in correspondence with the peri-implant bone due to the lack of control of the distribution of the masticatory load on the implants themselves. Although there is still no answer to the question of whether the incongruity of a prosthesis may or may not cause osseointegration failure, it is widely conceivable that the misfit of the implant-supported restoration has the intrinsic potential of causing implant failure. This failure is attributable both to the destruction of the bone-implant interface due to microfractures and ischemias and to mechanical failure due to the unscrewing or breaking of some prosthetic component. In fact, the screws that connect the meso and prosthetic superstructures, if not interfaced and perfectly coupled, can, once tightened, generate intense stresses (static stresses), which are of a considerably higher intensity than those generated during the patient's usual chewing function (dynamic stresses). These static loads are considered potentially more dangerous than dynamic loads, because they introduce permanent and incessant tensions in the peri-implant bone tissue, which, given the inelasticity of the bone-implant connection, is not able to dissipate them, as happens for the teeth, by means of orthodontic micro-movements (functional ankylosis) [21].

Therefore, although the long-term effects of these tensions on the stability and success of the implants and prostheses they support have not yet been fully understood, it can no doubt be assumed that the peri-implant bone does not benefit. Some authors argue that functional loads above a certain threshold, not yet specified, proportionally and progressively demolish the intimate bone-implant contact until a new homeostasis is reached. However, this reorganization of the tissues would not entail the new apposition of bone, but its replacement with fibrous connective tissue [22,23].

In 1996, Isidor, in a split-mouth study on monkeys, compared ligament peri-implantitis to occlusal overload. The results of this study led him to conclude that occlusal overload is the main risk factor for the loss of already osseointegrated implants, while the accumulation of bacterial plaque generally leads to marginal bone loss [24].

Other biomechanical variables can also affect the distribution of chewing forces on the fixture. These variables are:

- the quality and quantity of the bone;

- the length of the implant, its diameter, and its macro and microtopography;

- the number and position of the screws;

- the chosen prosthetic type, whether fixed or mobile, partial or total;

- the type of material chosen for the execution and the type of connection to the systems;

- and, finally, the type of patient occlusion, the presence of dysfunctions or parafunctions, muscle spasms, pre-contacts, or correct intermaxillary relationships.

To distribute the occlusal loads as evenly as possible and avoid transverse forces, the diameters and lengths of the implants should be the most desirable so as to transfer less stress to the peri-implant bone. Further measures suggest that the occlusal pillar should be below the centric cusp and the 
implant should be in the same direction as the force vector of the antagonist, but above all it is essential to finalize the centric with no interference in the eccentric lateral and protrusive movements.

\subsection{Passivation}

One of the factors most responsible for the generation of static stress inside the bone and on the peri-implant interface, in the absence of occlusal load, is represented by the lack of passive adaptation between the implant superstructure and the abutments.

Passive adaptation has been defined as "the circumferential and simultaneous contact of all the abutments on their respective implants and of all the gold cylinders of the prosthesis on their respective abutments"-i.e., the elimination of the tensions that develop in the structure when two or more systems are connected.

Some authors have described the complications, prosthetic and implant, due to the poor accommodation of the superstructures on the implants-i.e., the loosening or fracture of the implants or their prosthetic components (fixing screws and abutments) - in particular in prostheses screwed onto multiple implants, since in the cemented ones the cement would already partially cover the role of passivating agent. However, only a few studies have documented this causal link in a statistical way, above all by virtue of the fact that the deformations existing within the bone structures that surround implants that are not completely passivated derive from a combination of a series of variables such as tension forces, bending forces, and/or screwing. The main difficulties that prevent the achievement of a passive fit on the abutments are represented by the dimensional changes in the metal structure, especially related to casting and welding techniques, and/or the presence of impurities or micro-distortions on the adaptation surfaces after the over-casting of the cylinders or after the melting of the castable plastic standards.

Jemt et al. [25,26], in an experimental study on rabbit tibia, has shown that, in the absence of occlusal load, a discrepancy of $100 \mu \mathrm{m}$ in the 3-implant chaining structure can induce a bone-thickening reaction within a few weeks. Despite this, in light of other studies, they hypothesize that there must be a range of imprecision of superstructures tolerated by implants and compatible with their high long-term survival. The few investigations carried out on the biological effects on the peri-implant bone of the different degrees of marginal adaptation of the superstructures have shown that the imperfection limits of the metal reinforcements are not universally accepted, presenting values that are also different from each other.

\section{Results}

The physiological differences between a natural tooth and an endo-bone dental implant are well known, but the potential biomechanical characteristics derived from these differences remain controversial. The fundamental intrinsic difference is represented by the fact that osteointegrated implants are susceptible to occlusal overload, since they lack the periodontal ligament. While the mobility range of an osteointegrated implant has been shown to be approximately $3-5 \mu \mathrm{m}$, in a natural tooth the periodontal ligament adapts to occlusal forces with axial micromovements that can range between 25-100 $\mu \mathrm{m}$ (natural effect of "stress absorber"). The periodontal ligament, in fact, is functionally oriented perpendicular to the axial loads, allowing physiological movements of the tooth with respect to the occlusal stresses along its axis and a functional adaptation to changes in the conditions of static stress. Furthermore, this mobility allows it to functionally adapt to the deformations that the mandibular bone undergoes during opening or lateral movements.

Under load, the complex movements of a natural tooth initially involve the periodontal ligament and, in a secondary phase, the alveolar bone [27]. Vice versa, a loaded implant follows a linear deflection pattern, dependent on the elastic deformation of the alveolar bone. Thus, under load, the compressibility and deformability of the periodontal ligament of natural teeth can make a difference in adapting to static and dynamic occlusal forces compared to osseointegrated implants. 
A natural tooth, when subjected to a transverse load, is able to move rapidly by $56-108 \mu \mathrm{m}$ and rotate in the apical third of the root, and the lateral force borne by the tooth immediately tends to decrease from the bone crest along the root. On the other hand, the movement of an implant subjected to the same lateral load occurs gradually, reaching a maximum of $10-50 \mu \mathrm{m}$, and the concentration of forces is greater at the level of the crest of the peri-implant bone, with no possibility of the rotation of the implant. Richter [28] reported that the transverse load and the centric window frame develop the highest stresses on the crestal bone, and other studies likewise affirm that with greater loads the implants support them in correspondence with the marginal bone crest. Jacobs and Van Steenberghe [29], in a study in which they assessed the degree of occlusal perception of teeth and implants using occlusal interference, concluded that the pre-contact perceivable by natural teeth and implants, both in antagonism with natural teeth, is respectively 20 and $48 \mu \mathrm{m}$. In another study, by measuring oral tactile sensitivity with thin steel sheets, Mericske-Stern et al. [30] found that the minimum detectable pressure threshold was significantly higher in implants than in natural teeth (3.2 vs. 2.6 sheets). Similar results were also obtained by Keller and colleagues [31], in whose study the average perception threshold for implants was $100.6 \mathrm{~g}$, while that of natural teeth was $11.5 \mathrm{~g}$ (8.75 times lower).

Taylor believes that the loss of peri-implant crestal bone is the consequence of the plastic and elastic deformations of the supra-implant mechanical components rather than of the direction of the load. The same author, increasing the variables involved, maintains that the limiting factor is not the percentage of osseointegration, but the configuration of the implant and its elements, especially considering the long-term cyclical loads and, consequently, their distributions.

\subsection{Gnatological Implications in Implant Rehabilitations}

Four rules could be set that are the basis of implant and conventional rehabilitation (phenomena that aggravate TMJ (Temporo Mandibular Joint) disorders):

- $\quad$ steep incisive guide;

- decrease in the vertical dimension of the posterior sectors;

- $\quad$ steep eminence;

- increase in muscle activity.

The prosthesis of partially edentulous adult patients who already wear prostheses needs a preliminary diagnostic functional check to establish the condylar position and the vertical dimension. In the implant prosthesis, the patient with TMJ problems benefits from stable support given precisely by the implants, while a Kennedy class I rehabilitation with stable support can only improve the condylar situation.

The condylar pathologies of prosthetic origin can be reduced by packaging a prosthesis with the correct vertical position and condylar position, and also by reducing muscle tension and creating stable contact in the posterior region the condyles are brought back to a physiological position. The jaw behaves, during the work phase, as a third-degree lever. In the front or area of the guides, there is a greater displacement with a reduction in the surface load, the fulcrum is the articulation, and the resistance of the teeth means that the work of crushing the food bolus is carried out by the muscles, the masseter, and the internal and temporal pterygoid. During work and loading in the molar region, there can be a precontact that will be more harmful; it will be distal because the precontact will act as a fulcrum, transforming a third-class lever into a second-class one. If the disharmony exceeds the capacity of the adaptation of the disc, the condyle will come out of the usual seat, moving to the ventral position and creating an unbalanced situation. A suitable prosthetic result is obtained only after a functional investigation and a check of any pre-contacts. Muscle relaxation and the search for the hinge axis in a physiological position is the purpose of the decompression plate. 
The functional investigation begins with the taking of the impressions to pack the decompression plates; the necessary tools are an arbitrary transfer facial arch, a medium-value articulator, and the upper and lower models.

The functional investigation involves the preparation of the diagnostic functional temporaries. As a rule, in carrying out a rehabilitation the functional investigation, occlusion, vertical dimension, condylar position, and balanced occlusal load are of little importance. These important factors are usually not considered.

When waxing one or a few elements, it is good to reproduce the anatomy as faithfully as possible. When waxing an extended reconstruction, it is useful and important to know the vertical dimension and the values detected in the axiography. Repeatedly check the validity of the gnathological information with the provisionals, and finally with the occlusal replication. A universal concept teaches that it is useful to leave a degree of tolerance in eccentric movements.

Form is the plastic image of function, and the waxings close to natural canons adhere to this concept. The functional investigation uses two practical detection methods: With the rapid facial arch, suitable for medium-complexity jobs, the positioning of the upper jaw is obtained. With the kinematic arc, the Bennet is detected in the intercondylar axis (hinge). In complex cases, the kinematic arch is used for the construction of the medium and long-term provisional, and with the diagnostic provisional the validity of the detections is verified and the TMJ behavior is checked. Prudence advises us to reduce the occlusal surfaces in order to reduce the lever arm on the implant. The modern provisional has many roles-it is the therapeutic and diagnostic name. It is in the implant prosthesis that it finds its main application, and is often underestimated (Figure 3).
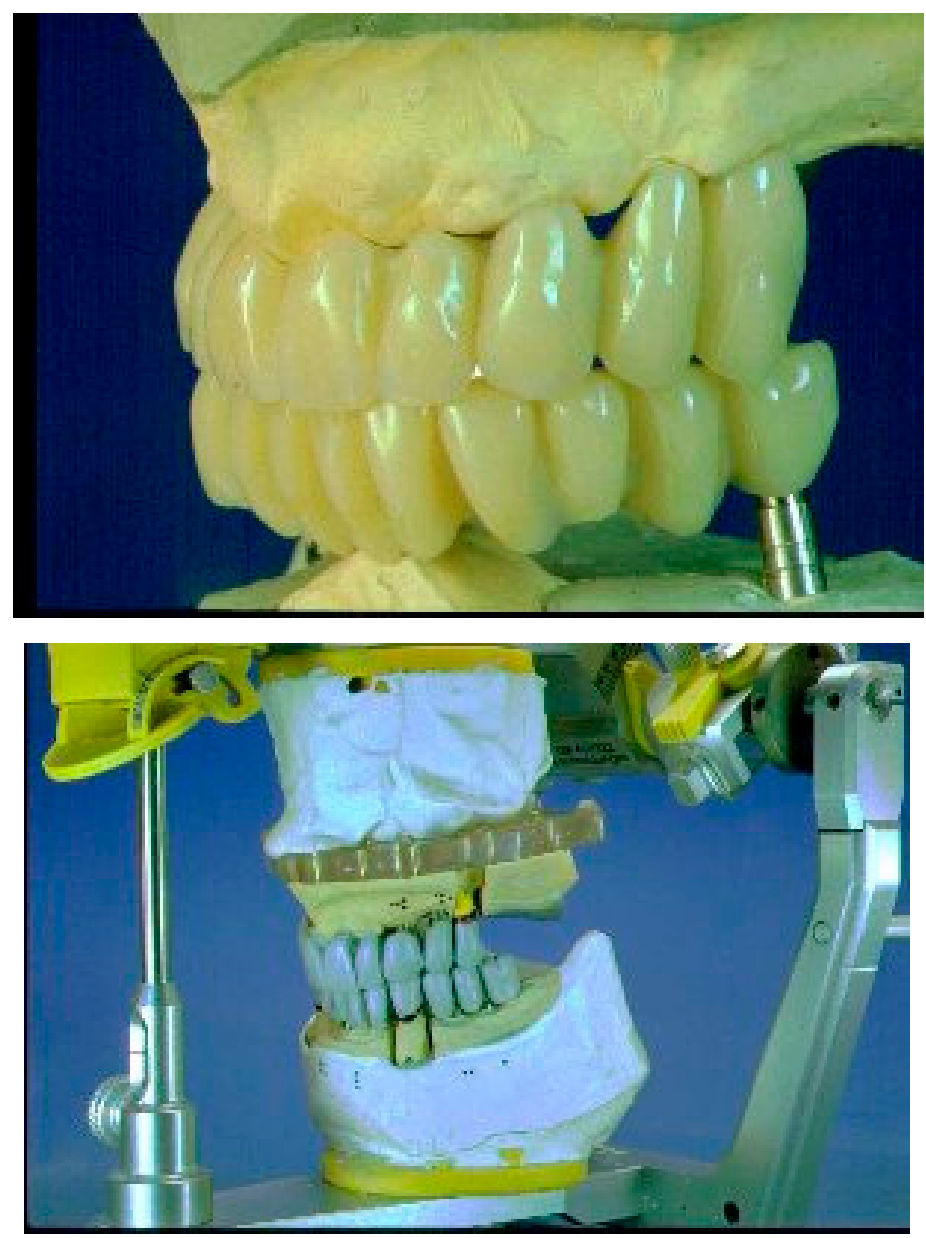

Figure 3. Occlusal line and test on articulator. 
The long-term diagnostic provisional is of enormous importance for the success of the final prosthesis. A design with good level characteristics must therefore be carried out: an axiographic investigation and a diagnostic wax-up on an individual articulator. In the search for the correct vertical dimension, the dentist can use the provisional to increase the vertical dimension; this method is based on the experience of the professional, who empirically determines the size. Alongside this, the radiologist and orthodontist determine on the basis angles and vertical dimension at different schools. The presence of any contractures determines a joint problem. During the medical history, the clinician will check the state of muscle tension, and a palpation to the muscles assigned to the occlusion will verify the pain. In the presence of problems at the A.T.M. before definitive rehabilitation, the clinician may decide to make small corrections to the occlusion; increase or decrease the vertical dimension; create guides; and bring the condyles back to the centric position, guiding the occlusion with an incisive canine guide and eliminating harmful contacts. The provisional diagnostic is responsible for the investigation and the functional verification before the definitive rehabilitation that will duplicate and follow it. Only after having ascertained the correct biomechanical functioning of the temporary bridge can the final ceramic metal girder of the occlusal line be realized $[32,33]$.

\subsection{Clinical Case}

Patient: A.G., age 57.

Clinical examination: the intraoral examination shows an absence of 2.6 and a fixed prosthesis in the area of 2.3-2.5.

Intraoral X-ray examination: the lack of integrity and inconsistency of 2.3 and 2.5 (pillar elements of the fixed prosthesis) are highlighted (Figure 4).

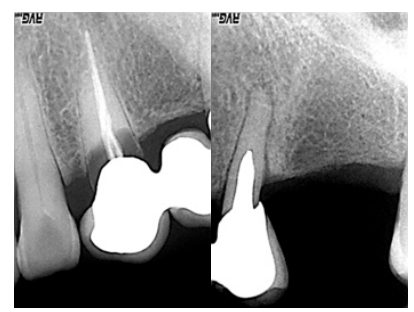

Figure 4. Preliminary radiographs.

In consideration of the clinical evaluations that prevented the conservative restoration of the bridge pillars and the instrumental examination, a treatment was planned that involved the extraction of 2.3 and 2.5 and an implant-prosthetic rehabilitation of the elements 2.3, 2.4, 2.5, and 2.6.

Clinical diary:

About 40 days after the extraction of the dental elements, the insertion of four implants is performed.

Six months after the insertion of the implants, an operation was performed to uncover the implants and mount the healing abutments. A few weeks later, the impression was taken by means of an individual tray with the pick-up technique (Figures 5 and 6 ).

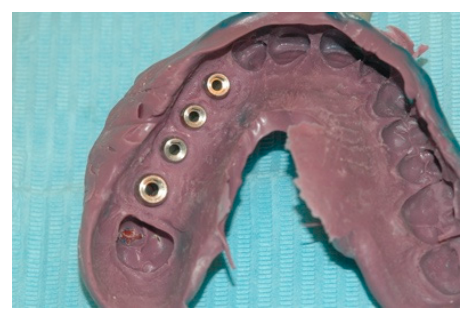

Figure 5. Impression and structure made. 


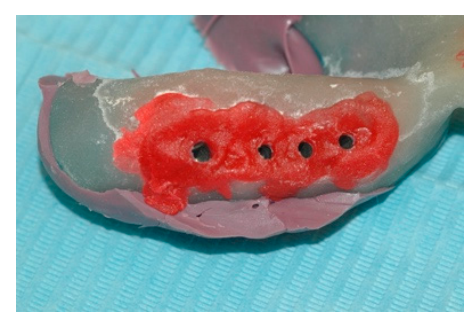

Figure 6. Impression and structure made.

As a prosthetic solution, a bridge of four metal-ceramic elements cemented on a screwed mesostructure has been provided.

On the basis of the impression taken, the model was developed and a wax structure was created according to the prosthetic planning and the position of the implants on a model developed with removable soft gingiva (Figure 7).

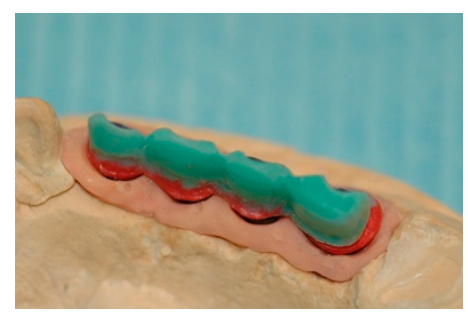

Figure 7. Impression and structure made.

According to the procedures established for the NobelProcera ${ }^{\circledR}\left(\right.$ Nobel Biocare ${ }^{\circledR}$, Zurich, Switzerland) system used in the resolution of this clinical case, the structure was scanned using CAD/CAM, which was then sent in electronic format to the Swedish parent company. The next operational step involved the construction of a mesostructure made by milling from solid, with numerically controlled industrial machinery that represents a copy of our metal structure.

Once the mesostructure was made, it was checked to verify passive adaptation and its precise coupling with the arched fixtures (Figures 8 and 9).

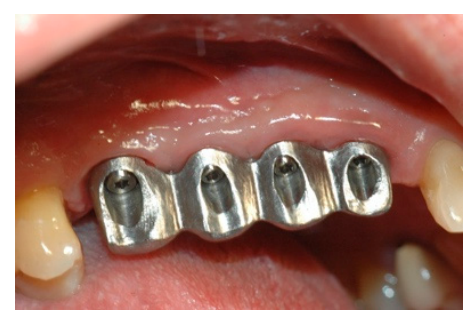

Figure 8. Mesostructure test.

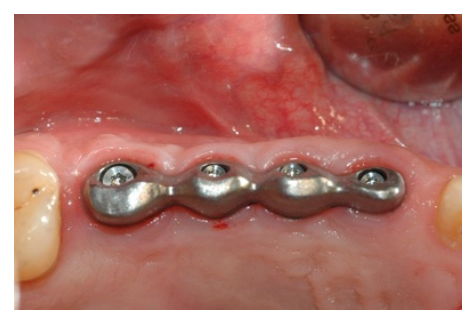

Figure 9. Mesostructure test.

Upon positive feedback, the construction of a superstructure was carried out by performing fusion and subsequent ceramicization in the laboratory (Figures 10 and 11). 


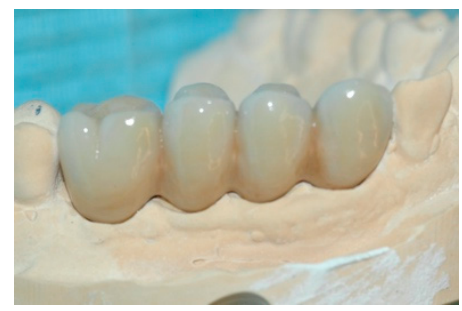

Figure 10. The built superstructure.

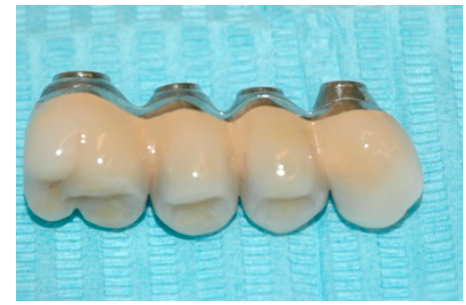

Figure 11. The built superstructure.

When the prosthesis was positioned, the mesostructure was fixed by tightening the screws with a torque wrench at $30 \mathrm{Ncm}$, and the superstructure was cemented by carrying out a radiographic check to evaluate the structural coherence (Figures 12-14).

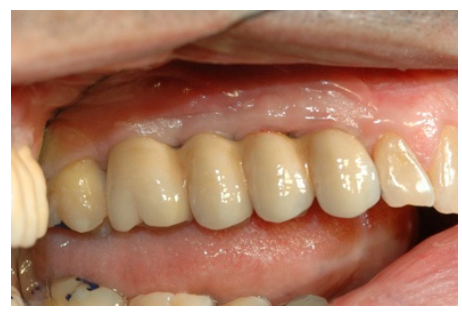

Figure 12. Cementing the superstructure.

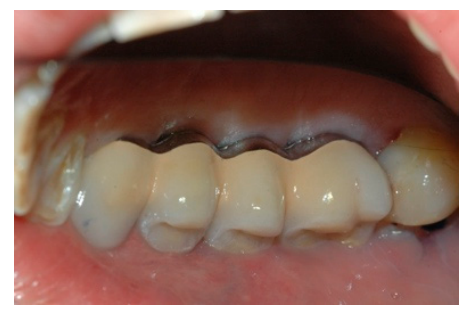

Figure 13. Cementing the superstructure.

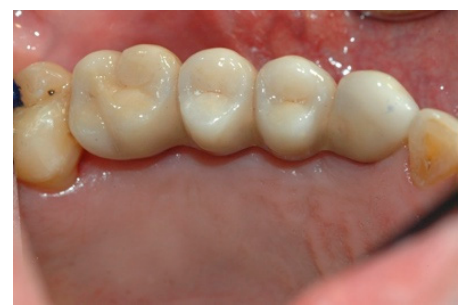

Figure 14. Cementing the superstructure.

\section{Discussion}

The purpose of passivation techniques is to ensure the best possible biomechanical functioning of implant prosthetic rehabilitation, considering that controllable and foreseeable adaptation is essential 
to prevent possible mechanical complications. Jemt et al. studies have shown that, in cases of total edentulism, there is a certain biological tolerance of the live bone for the maladjustments of the prosthesis which determine the static forces and can be stimulating for the peri-implant bone, inducing positive bone remodeling [25,26,34-41].

However, this finding is valid if these static forces deriving from the failure of passivation do not combine with further defined dynamic stresses deriving from abnormal occlusal load. However, there is evidence in the literature that the lack of good adaptation of the superstructure on the implants leads to an increased risk of the unscrewing and/or fracturing of the mechanical components.

This risk, added to disproportionate or non-axial occlusal loads, probably underlies the failure of the implants that support structures that are not adequately passivated. This hypothesis seems compatible with the "mechanostatic" theory developed by Frost in 1983 [42,43], which states that the bone reacts to the load according to the mechanical laws of induced deformation, and identifies five load thresholds, all measured in microstrain (keep in mind that $1 \%$ of deformation corresponds to 10,000 microstrain):

1. disuse atrophy threshold: between 0 and 200, the interval in which the bone undergoes a reduction in its mass;

2. remodeling threshold: between 200 and 2500, a range in which the bone maintains its structure and mass unaltered;

3. hypertrophy threshold: between 2500 and 4000 , a condition in which the bone tissue reacts to the load by increasing its mass and modifying its structure;

4. overload threshold: above 4000 , the limit above which the bone tends to reabsorb due to the formation of micro-cracks, which would induce bone repair and weakening phenomena;

5. acute breaking load: between 10,000 and 25,000 , the threshold above which the bone fractures.

The need for the passivation phase arises mainly from the dimensional alteration of the adaptation of the metal reinforcement to the systems linked to the realization of the working model. In fact, the impression is the main means of communication of the clinical situation between the study and the laboratory, with the aim of transmitting all the information necessary for the realization of the prosthetic product in the most precise way possible. It represents the crucial verification of all the preparatory work prior to the patient's prosthetic rehabilitation, having to provide the correct reading of the position of the implants to try to "absorb" all the other potential, non-controllable errors in the construction process that could invalidate the "perfect" fit of metal superstructures on the abutments. In implant prosthesis, these steps are of particular importance considering the fact that the osseointegrated fixture has a high degree of rigidity and a low resilience, characteristics which, under the purely prosthetic profile, unfortunately translate into a defect, requiring extremely precise artefacts both in the components and anatomy to evenly distribute chewing loads and ensure minimal resilience.

Generally, to determine the position of the fixtures, false transfer abutments are used, which must be screwed to the fixtures themselves, and can remain incorporated in the impression or be subsequently repositioned in it, depending on whether the pick-up technique is used or the traditional one. The first, although more laborious, is preferable due to the great precision that the individual perforated spoon guarantees, from which the transfer fixing screws must protrude once the impression tray is positioned in the patient's oral cavity in order to be unscrewed and removed together with the spoon. The second method, on the other hand, despite being quick to perform, can lead to errors during the repositioning of the transfers in the impression. In both cases, however, it is necessary to have materials with a good dimensional stability, limited thermal contraction, limited hardening contraction, great rigidity, excellent elastic memory, and hydrophilicity, and that are capable of being distributed on all metal components, capable of undergoing small mistreatments deriving from the need to unscrew the impression transfers not yet removed, or capable of accepting them with precision if the second method is used. 
The same expertise that is required of the dentist must also characterize the work of the dental technician, who will have to pay the utmost attention to every single processing phase, from the preparation of the plaster models to the waxing, coating, casting, and welding. Although these processing procedures are carried out with extreme control, both by the operator and the industry, there are, however, variables that, if considered individually, would be dimensionally irrelevant, but added together make the clinical realities different from those of the laboratory. They generate sometimes imperfect prosthetic products that are capable of producing tensions, which could be the cause of innumerable implant prosthetic failure factors, including unscrewing the connection screws, the fracture of the stumps or of the armature, bone resorption, peri-implantitis, or even the loss of the system itself.

Ultimately, whatever the method used, what matters is the result-that is, a superstructure that is as free of tension as possible. It is, therefore, fundamental to know the problem and face it from time to time according to the operations that are most suitable for the clinician and technician.

This technology uses electronically controlled milling to obtain titanium mesostructures that are as passive as possible with the one-piece subtraction technique. This technique, according to the data published by the manufacturer, has proven to be valid, since it allows us to minimize the static forces (considered potentially the most dangerous for the survival of rehabilitations on implants) both between the prosthetic components and inside the bone tissue. The manufacturer himself guarantees a marginal gap of around $20 \mu \mathrm{m}$ on the vertical plane and around $30 \mu \mathrm{m}$ on the horizontal plane.

In any case, resorting to solutions of this type becomes, therefore, practically indispensable, because they allow us, with relative simplicity, to obtain metal mesostructures characterized by a precise marginal closure and by the absence of static forces on the components.

It is clear that the success of each technique is mainly linked to its constant repeatability. Being able to improve mesostructures and predict biomechanical forces through digital design could be a goal to set in order to limit short, medium, and long-term rehabilitation failures. The clinical case has been carried out as an example of a technique that allows us, with relative ease and less waste of time and contained materials, to create a prosthetic device with standardized and controlled techniques that determines a significant reduction in failures.

\section{Conclusions}

The main defect of this technique for the realization of a total or removable prosthesis on implants is that of requiring an accurate preliminary analysis of the intermaxillary ratios and an extension of the time of the realization of the prosthesis, especially if the assembly of the artificial teeth to the correct vertical dimension entails, from part of the surgeon, additive surgery on the bone that requires complete surgical healing; in exchange, you have the possibility of knowing with certainty, from the beginning, what the finished result will be, in harmony with the absolute determinants of the quality of a definitive total prosthesis. Knowing and predicting the discharge of mechanical forces through prosthetic structures and implants can help the clinician in limiting the short and long-term complications of this type of rehabilitation.

Author Contributions: Conceptualization, C.D.; writing-original draft preparation, C.D.; writing-review and editing, S.B. and C.S.; supervision, S.S.; visualization, G.S.; project administration, L.F. All authors have read and agreed to the published version of the manuscript.

Funding: This research received no external funding.

Conflicts of Interest: The authors declare no conflict of interest. 


\section{References}

1. Branemark, P.I.; Adell, R.; Breine, U.; Hansson, B.O.; Lindstrom, J.; Ohlsson, A. Intra-osseous anchorage of dental prostheses. I. Experimental studies. Scand. J. Plast. Reconstr. Surg. 1969, 3, 81-100. [CrossRef] [PubMed]

2. Taylor, T.D.; Wiens, J.; Carr, A. Evidence-based considerations for removable prosthodontic and dental implant occlusion: A literature review. J. Prosthet. Dent. 2005, 94, 555-560. [CrossRef] [PubMed]

3. Katona, T.R.; Eckert, G.J. The mechanics of dental occlusion and disclusion. Clin. Biomech. 2017, 50, 84-91. [CrossRef] [PubMed]

4. Gong, P. Role of occlusion in complications of implant rehabilitation. Zhonghua Kou Qiang Yi Xue Za Zhi 2018, 53, 800-804. [CrossRef]

5. Fabbri, G.; Sorrentino, R.; Cannistraro, G.; Mintrone, F.; Bacherini, L.; Turrini, R.; Bombardelli, T.; Nieri, M.; Fradeani, M. Increasing the Vertical Dimension of Occlusion: A Multicenter Retrospective Clinical Comparative Study on 100 Patients with Fixed Tooth-Supported, Mixed, and Implant-Supported Full-Arch Rehabilitations. Int. J. Periodontics Restor. Dent. 2018, 38, 323-335. [CrossRef]

6. Stanford, C.M. Issues and considerations in dental implant occlusion: What do we know, and what do we need to find out? J. Calif. Dent. Assoc. 2005, 33, 329-336.

7. Sheridan, R.A.; Decker, A.M.; Plonka, A.B.; Wang, H.L. The Role of Occlusion in Implant Therapy: A Comprehensive Updated Review. Implant. Dent. 2016, 25, 829-838. [CrossRef]

8. Orjonikidze, Z.; Orjonikidze, R.; Arutyunov, S. Peculiarities of the Occlusion Formation in Dental Implant Supported Artificial Teeth. Georgian Medical News, 1 January 2018; 24-30.

9. Li, X.Q.; Ma, X.N.; Xu, X. Occlusal analysis of implant-supported fixed denture in edentulous patients. Hua Xi Kou Qiang Yi Xue Za Zhi 2018, 36, 628-632. [CrossRef]

10. Schnitman, P.A.; Wohrle, P.S.; Rubenstein, J.E. Immediate fixed interim prostheses supported by two-stage threaded implants: Methodology and results. J. Oral Implantol. 1990, 16, 96-105.

11. Branemark, P.I.; Hansson, B.O.; Adell, R.; Breine, U.; Lindstrom, J.; Hallen, O.; Ohman, A. Osseointegrated implants in the treatment of the edentulous jaw. Experience from a 10-year period. Scand. J. Plast. Reconstr. Surg. Suppl. 1977, 16, 1-132.

12. Rizzo, S.; Zampetti, P.; Rodriguez Y Baena, R.; Svanosio, D.; Lupi, S.M. Retrospective analysis of 521 endosseous implants placed under antibiotic prophylaxis and review of literature. Minerva Stomatol. 2010, 59, 75-88. [PubMed]

13. Lupi, S.M.; Zaffe, D.; Baena, R.R.Y.; Rizzo, S.; Botticelli, A.R. Cytopathological and chemico-physical analyses of smears of mucosa surrounding oral piercing. Oral Dis. 2010, 16, 160-166. [CrossRef]

14. Lupi, S.M.; Granati, M.; Butera, A.; Collesano, V.; Rodriguez Y Baena, R. Air-abrasive debridement with glycine powder versus manual debridement and chlorhexidine administration for the maintenance of peri-implant health status: A six-month randomized clinical trial. Int. J. Dent. Hyg. 2017, 15, 287-294. [CrossRef] [PubMed]

15. Xueyang, Z.; Yanhong, H.; Pei, C.; Pan, J.; Yarong, W.; Yuan, S. Occlusion design of edentulous implant prosthesis. Hua Xi Kou Qiang Yi Xue Za Zhi 2018, 36, 1-3. [CrossRef]

16. Szmukler-Moncler, S.; Salama, H.; Reingewirtz, Y.; Dubruille, J.H. Timing of loading and effect of micromotion on bone-dental implant interface: Review of experimental literature. J. Biomed. Mater. Res. 1998, 43, 192-203. [CrossRef]

17. Stegaroiu, R.; Sato, T.; Kusakari, H.; Miyakawa, O. Influence of restoration type on stress distribution in bone around implants: A three-dimensional finite element analysis. Int. J. Oral Maxillofac. Implant. 1998, 13, 82-90.

18. Stegaroiu, R.; Kusakari, H.; Nishiyama, S.; Miyakawa, O. Influence of prosthesis material on stress distribution in bone and implant: A 3-dimensional finite element analysis. Int. J. Oral Maxillofac. Implant. 1998, 13, 781-790.

19. Misch, C.M. Bur guide surgical template for implant placement in grafted jaws. J. Oral Implantol. 1997, 23, 170-173.

20. Tarnow, D.P.; Emtiaz, S.; Classi, A. Immediate loading of threaded implants at stage 1 surgery in edentulous arches: Ten consecutive case reports with 1- to 5-year data. Int. J. Oral Maxillofac. Implant. 1997, 12, 319-324.

21. Cawood, J.I.; Howell, R.A. A classification of the edentulous jaws. Int. J. Oral Maxillofac. Surg. 1988, 17, 232-236. [CrossRef] 
22. Ilizarov, G.A. The tension-stress effect on the genesis and growth of tissues. Part I. The influence of stability of fixation and soft-tissue preservation. Clin. Orthop. Relat. Res. 1989, 238, 249-281. [CrossRef]

23. Ilizarov, G.A. The tension-stress effect on the genesis and growth of tissues: Part II. The influence of the rate and frequency of distraction. Clin. Orthop. Relat. Res. 1989, 239, 263-285. [CrossRef]

24. Isidor, F. Loss of osseointegration caused by occlusal load of oral implants. A clinical and radiographic study in monkeys. Clin. Oral Implant. Res. 1996, 7, 143-152. [CrossRef] [PubMed]

25. Jemt, T.; Book, K. Prosthesis misfit and marginal bone loss in edentulous implant patients. Int. J. Oral Maxillofac. Implant. 1996, 11, 620-625.

26. Jemt, T.; Lekholm, U.; Johansson, C.B. Bone response to implant-supported frameworks with differing degrees of misfit preload: In vivo study in rabbits. Clin. Implant. Dent. Relat. Res. 2000, 2, 129-137. [CrossRef]

27. Parfitt, G.J. Measurement of the physiological mobility of individual teeth in an axial direction. J. Dent. Res. 1960, 39, 608-618. [CrossRef]

28. Richter, E.J. In vivo horizontal bending moments on implants. Int. J. Oral Maxillofac. Implant. 1998, 13, 232-244.

29. Jacobs, R.; van Steenberghe, D. Comparison between implant-supported prostheses and teeth regarding passive threshold level. Int. J. Oral Maxillofac. Implant. 1993, 8, 549-554.

30. Mericske-Stern, R.; Assal, P.; Mericske, E.; Burgin, W. Occlusal force and oral tactile sensibility measured in partially edentulous patients with ITI implants. Int. J. Oral Maxillofac. Implant. 1995, 10, 345-353. [CrossRef]

31. Keller, D.; Hammerle, C.H.; Lang, N.P. Thresholds for tactile sensitivity perceived with dental implants remain unchanged during a healing phase of 3 months. Clin. Oral Implant. Res. 1996, 7, 48-54. [CrossRef]

32. Cardonnet, M.; Clauzade, M. Differential diagnosis of TMJ dysfunctions. Cah. Prothes. 1987, 15, 125-170.

33. Choi, B.H.; Yi, C.K.; Yoo, J.H. MRI examination of the TMJ after surgical treatment of condylar fractures. Int. J. Oral Maxillofac. Surg. 2001, 30, 296-299. [CrossRef] [PubMed]

34. Jemt, T.; Lekholm, U. Implant treatment in edentulous maxillae: A 5-year follow-up report on patients with different degrees of jaw resorption. Int. J. Oral Maxillofac. Implant. 1995, 10, 303-311.

35. Jemt, T.; Lekholm, U. Measurements of bone and frame-work deformations induced by misfit of implant superstructures. A pilot study in rabbits. Clin. Oral Implant. Res. 1998, 9, 272-280. [CrossRef]

36. Cicciù, M.; Cervino, G.; Terranova, A.; Risitano, G.; Raffaele, M.; Cucinotta, F.; Santonocito, D.; Fiorillo, L. Prosthetic and Mechanical Parameters of the Facial Bone under the Load of Different Dental Implant Shapes: A Parametric Study. Prosthesis 2019, 1, 41-53. [CrossRef]

37. Cervino, G.; Montanari, M.; Santonocito, D.; Nicita, F.; Baldari, R.; De Angelis, C.; Storni, G.; Fiorillo, L. Comparison of Two Low-Profile Prosthetic Retention System Interfaces: Preliminary Data of an In Vitro Study. Prosthesis 2019, 1, 54-60. [CrossRef]

38. Scrascia, R.; Fiorillo, L.; Gaita, V.; Secondo, L.; Nicita, F.; Cervino, G. Implant-Supported Prosthesis for Edentulous Patient Rehabilitation. From Temporary Prosthesis to Definitive with a New Protocol: A Single Case Report. Prosthesis 2020, 2, 10-24. [CrossRef]

39. Fiorillo, L.; D'Amico, C.; Turkina, A.Y.; Nicita, F.; Amoroso, G.; Risitano, G. Endo and Exoskeleton: New Technologies on Composite Materials. Prosthesis 2020, 2, 1-9. [CrossRef]

40. Ortensi, L.; Vitali, T.; Bonfiglioli, R.; Grande, F. New Tricks in the Preparation Design for Prosthetic Ceramic Laminate Veeners. Prosthesis 2019, 1, 29-40. [CrossRef]

41. Cicciù, M. Prosthesis: New Technological Opportunities and Innovative Biomedical Devices. Prosthesis 2019, 1, 1-2. [CrossRef]

42. Frost, H.M. A determinant of bone architecture. The minimum effective strain. Clin. Orthop. Relat. Res. 1983, 175, 286-292.

43. Frost, H.M. The skeletal intermediary organization. Metab. Bone Dis. Relat. Res. 1983, 4, 281-290. [CrossRef]

(C) 2020 by the authors. Licensee MDPI, Basel, Switzerland. This article is an open access article distributed under the terms and conditions of the Creative Commons Attribution (CC BY) license (http://creativecommons.org/licenses/by/4.0/). 